\title{
Activity and safety of sunitinib in poor risk metastatic renal cell carcinoma patients
}

Romualdo Barroso-Sousa', Rodrigo R. Munhoz',2, Milena P. Mak', Leonardo G. Fonseca', Angelo B. S. Fede ${ }^{1}$, Rudinei Diogo Marques Linck², Clovis R. Coelho ${ }^{3}$, Camila M. V. Moniz', Ciro E. Souza ${ }^{1,2}$, Carlos Dzik',2

${ }^{1}$ Instituto do Câncer do Estado de São Paulo, Faculdade de Medicina, Universidade de São Paulo, São Paulo, Brazil; 2Centro de Oncologia, Hospital Sírio-Libanês, São Paulo, Brazil; 3Departament of Radiology and Oncology, Faculdade de Medicina, Universidade de São Paulo, São Paulo, Brazil

\section{ABSTRACT}

Purpose: To assess the activity, safety and treatment patterns of sunitinib in patients with poor-risk metastatic renal cell carcinoma (mRCC).

Materials and Methods: We retrospectively reviewed the charts of poor risk patients treated with sunitinib from October 2006 to July 2013 who met the eligibility criteria. The primary endpoint was overall survival (OS). Tumor radiological response was measured according to RECIST 1.1 and adverse events (AEs) were assessed through standard criteria.

Results: Median OS was 8.16 months (95\% CI, 5.73-10.59). Of the 53 patients included in this analysis, $9(17.0 \%)$ achieved partial response, 12 (22.6\%) had stable disease. Median treatment duration was 3.30 months (95\% CI: 1.96-4.63) and 26.4\% of patients discontinued treatment due to toxicity. Grade 3 or higher AEs occurred in 39.6\% of patients, the most common being fatigue (15.1\%), neutropenia (9.5\%), nausea, vomiting and diarrhea (7.5\% each).

Discussion: Sunitinib may benefit some unselected poor-risk patients, although the rates of AEs and drug discontinuation suggest a need for careful patient monitoring.

\section{ARTICLE INFO}

\section{Key words: sunitinib [Supplementary Concept]; Carcinoma, Renal Cell; Safety;}

Int Braz J Urol. 2014; 40: 835-41

\section{Submitted for publication:}

February 05, 2014

\section{Accepted after revision:}

May 03, 2014

\section{INTRODUCTION}

Over the past few years, several new agents have been granted approval for the first-line treatment of metastatic renal cell carcinoma (mRCC). These agents include inhibitors of the vascular endothelial growth factor (VEGF) pathway (sunitinib, pazopanib, bevacizumab) and inhibitors of the mammalian target of rapamycin (mTOR) pathway (temsirolimus) (1).

With the exception of the trial investigating temsirolimus (2), pivotal large randomized phase III clinical trials supporting the use of these medications in mRCC excluded or underrepresented patients with poor risk features. However, the efficacy and safety of targeted therapies such as sunitinib in this population is less clear (3). Recently published data suggests that at least 30\% of mRCC patients receiving VEGF pathway inhibitors belong to the poor-risk group based on the International Database Consortium (IDC) prognostic model (4). Therefore, we performed a retrospective analysis to evaluate the efficacy, safety and treatment patterns of sunitinib in a non-selected population of poor-risk mRCC patients. 


\section{MATERIALS AND METHODS}

\section{Study design and population}

This study was a retrospective analysis of metastatic RCC patients' electronic charts. All patients were treated with sunitinib at two major cancer centers located in the city of São Paulo, Brazil (Instituto do Câncer do Estado de São Paulo, Universidade de São Paulo and Centro de Oncologia, Hospital Sirio-Libanês), between October 2009 and July 2013. Patients were identified from the hospitals' administrative databases, and relevant information was retrieved from electronic medical records. This analysis was approved by the Institutional Review Boards of both institutions.

The primary study objective was to determine the population's overall survival (OS). Secondary objectives were the following: examination of the safety profiles, frequency of treatment modifications, time to treatment discontinuation and response rate in an outside clinical trial setting.

The patient inclusion criteria were (a) diagnosis of metastatic RCC of any histologic type; (b) poor risk features according to the IDC model (4); (c) a Karnofsky performance status (KPS) of 60 or more at baseline assessment and (d) anti-VEGF-naïve patients before starting sunitinib.

\section{Assessment}

Trained physicians extracted patients treatment data from electronic medical records, including the following information: date of RCC diagnosis, demographic variables, comorbidities, metastatic site(s), baseline KPS, drug-related AE data, laboratory data, and the results of key radiological tests. Additionally, the first and last dates of sunitinib use, treatment modifications, and both baseline and follow-up tumor measurements were also recorded. A patient's observation period began on the date of initiation of sunitinib and ended either at the time of their last center visit or death.

\section{Outcome Definitions}

Safety

Safety outcomes included the numbers and proportion of patients who experienced spe- cific adverse events (AE), of any grade and of grade 3 or higher. We retrospectively assessed AE and assigned grade levels based on the National Cancer Institute's Common Terminology Criteria for Adverse Events (CTCAE), version 3.0, at chart abstraction because grade levels of adverse events are not regularly recorded in medical charts in clinical settings (5).

\section{Treatment Patterns}

Treatment patterns included the numbers and proportion of patients who discontinued treatment or had dose modifications during sunitinib treatment and those who switched to a second-line treatment. The type, date, and the reasons for treatment modification were obtained from the patients' medical charts. Time to treatment discontinuation (TTD) was defined as the time from starting sunitinib to the date of the last administered dose independent of cause for discontinuation or loss to follow-up, whichever occurred first.

\section{Efficacy}

Overall survival was defined as the interval between the start of therapy and death from any cause (with survival times censored at last follow-up for patients alive at the time of last assessment). The response assessment was reviewed by an independent radiologist, using guidelines for change in the sum of maximal diameters as defined by Response Evaluation Criteria In Solid Tumors, but responses were not confirmed by a second assessment (6).

\section{Statistical analysis}

The patient baseline characteristics, overall response rate (ORR), AEs and sunitinib treatment patterns were reported based on descriptive statistics. Means, medians and ranges were used to describe continuous variables, and frequencies or percentages were used for categorical variables. TTD and OS were estimated by the Kaplan-Meier method, and their 95\% confidence interval (CI) were calculated. All data analyses were conducted using SPSS, version 21.0, Armonk, NY. 


\section{RESULTS}

\section{Patients}

Fifty-three eligible patients were identified. Patients' demographic and clinical characteristics are shown in Table-1. The median age was 61.6 years (range 31.2-89.6). The proportion of patients with 3, 4, and 5 adverse prognosis factors were $62.3 \%, 18.9 \%$ and 18.9\%, respectively. Most patients were male (71.7\%) and had clear-cell histo$\log$ (90.6\%) with no previous systemic treatment

Table 1 - Patient characteristics.

\begin{tabular}{lc}
\hline Characteristics & $\begin{array}{c}\text { Number of patients } \\
(\mathbf{n}=53)\end{array}$ \\
\hline Age (years) & \\
median (range) & $61.6(31.2-89.6)$ \\
Sex, $\mathbf{n}(\%)$ & \\
Male & $38(71.7 \%)$ \\
Female & $15(28.3 \%)$ \\
KPS, $\mathbf{n}$ (\%) & \\
$\geq 80$ & $25(47.2 \%)$ \\
60-70 & $28(52.8 \%)$ \\
Histology, $\mathbf{n}$ (\%) & \\
Clear cell & $48(90.6 \%)$ \\
Other & $5(9.4 \%)$ \\
Previous nephrectomy, $\mathbf{n}$ (\%) & \\
Yes & $30(56.6 \%)$ \\
No & $23(43.4 \%)$ \\
Previous systemic therapy, $\mathbf{n}(\%)$ & \\
None & $47(88.7 \%)$ \\
Immunotherapy & $3(5.7 \%)$ \\
Target therapy & $3(5.7 \%)$ \\
Number of metastatic sites, $\mathbf{n}(\%)$ & \\
1 & $9(17.0 \%)$ \\
2 & $20(37.7 \%)$ \\
$>2$ & $24(45.3 \%)$ \\
Sites of metastases, $\mathbf{n}$ (\%) & \\
Lung & \\
Lymph nodes & $40(75.5 \%)$ \\
Bone & $25(47.2 \%)$ \\
Liver & $21(39.6 \%)$ \\
Brain & $15(28.3 \%)$ \\
Other & $4(7.5 \%)$ \\
\hline & $22(41.5 \%)$ \\
\hline
\end{tabular}

KPS: Karnofsky performance status.
(88.7\%). As expected, other common poor prognosis features were found in the population. These features included KPS of $\leq 70$ (52.8\%), no previous nephrectomy (43.4\%) and three or more metastatic sites at baseline (45.3\%) (Table-1). The most prevalent metastatic sites were the lungs (75.5\%), followed by lymph nodal involvement (47.2\%).

\section{Treatment patterns}

Table-2 summarizes the sunitinib treatment patterns and reasons for discontinuation. Thirty seven patients $(69.8 \%)$ started on therapy at the standard schedule of $50 \mathrm{mg}$ once daily, 4 weeks on, followed by 2 weeks off; sixteen patients (30.2\%) started treatment at a reduced dose. Although not statistically significant, patients on standard regimen were younger (median age: 58.6 versus 68.8 years; $p=0.1$ ) than those on reduced dose regimens. Median TTD was 3.30 months (95\% CI: 1.96-4.63). At the time of analysis, almost all patients had discontinued therapy (96.2\%), mostly due to disease progression (69.8\%). Drug toxicity caused treatment interruption in $26.4 \%$ of patients. Furthermore, adverse events required dose modifications in $26.4 \%$ of patients. Three patients (5.7\%) experienced dose escalation after starting treatment at a reduced dose.

\section{Safety}

Adverse events reported in patients' medical charts are summarized in Table-3. Among patients included in this retrospective analysis, 94.3\% experienced at least one AE, including grade 3 or higher AE. The most common all-grade $\mathrm{AE}$ was fatigue or asthenia (64.1\%), followed by nausea $(49.0 \%)$, stomatitis $(41.5 \%)$, vomiting $(37.7 \%)$ and hypothyroidism (32.1\%). Grade 3 or higher AE was reported in 39.6\% of patients. The most common grade 3 or higher $\mathrm{AE}$ were fatigue (15.1\%), neutropenia (9.5\%), nausea, vomiting, and diarrhea (7.5\% each). There was one treatment-related death due to febrile neutropenia and septic shock. There was no statistically significant difference in the frequency of grade 3 or higher AEs between patients who started sunitinib at the standard dose and patients who initiated therapy at a reduced dose regimen. 
Table 2 - Sunitinib treatment patters.

Number of patients $(n=53)$

\begin{tabular}{lc}
\hline Initial dose & $37(69.8 \%)$ \\
- 50mg QD 4weeks on 2 off & $16(30.2 \%)$ \\
$\quad$ - Reduced dose & \\
Duration of treatment, months & $3.30(1.96-4.63)$ \\
- Median (95\% CI) & $14(26.4 \%)$ \\
Patients with sunitinib dose reduction & $37(69.8 \%)$ \\
Status of sunitinib treatment at time of analysis & $14(26.4 \%)$ \\
- Discontinuation due to disease progression & $2(3.8 \%)$ \\
- Discontinuation due to adverse events & \\
\hline
\end{tabular}

Cl: confidence interval; QD: once daily.

Table 3 - Reported adverse events during sunitinib treatment* .

\begin{tabular}{lcc}
\hline Adverse event & All grades, $\mathrm{n}(\%)$ & Grades $3 / 4, \mathrm{n}(\%)$ \\
\hline Any & $50(94.3)$ & $21(39.6)$ \\
Fatigue/asthenia & $34(64.1)$ & $8(15.1)$ \\
Nausea & $26(49.0)$ & $4(7.5)$ \\
Stomatitis & $22(41.5)$ & $3(5.7)$ \\
Vomiting & $20(37.7)$ & $4(7.5)$ \\
Hypothyroidism & $17(32.1)$ & $2(3.8)$ \\
Anemia & $12(22.6)$ & $1(1.9)$ \\
Hand-foot syndrome & $12(22.6)$ & $3(5.7)$ \\
Hypertension & $10(18.8)$ & - \\
Diarrhea & $9(16.8)$ & $4(7.5)$ \\
Neutropenia & $7(13.3)$ & $5^{+}(9.5)$ \\
Thrombocytopenia & $7(13.3)$ & $2(3.8)$ \\
\hline
\end{tabular}

*Adverse events experienced by at least $10 \%$ of patients are reported; +Including a grade 5 adverse event.

\section{Efficacy}

Of the 53 patients included in this analysis, nine (17.0\%) achieved partial response, 12 (22.6\%) had stable disease and 13 (24.5\%) had progressive disease. Nineteen patients (35.8\%) were not evaluated for response, including 14 patients
(26.4\%) with early discontinuation: one treatment-related death caused by febrile neutropenia; one loss to follow-up before response evaluation; and $4(7.5 \%)$ cases of early discontinuation due to limiting AE. No complete responses were seen. At a median follow-up of 7.5 months, 9 patients 
(16.9\%) were still alive. The estimated median OS was 8.16 months (95\% CI, 5.73-10.59) (Figure-1). There was no statistically significant difference in median OS between patients who started sunitinib at the standard dose (9.1 months; 95\% CI, 6.1212.07) and patients with modified regimens (7.2 months; 95\% CI, 6.28-8.11). Only 6 patients received a subsequent treatment after discontinuing sunitinib (five of them were treated with interferon-alpha and one was treated with everolimus).

\section{DISCUSSION}

Data regarding the use of sunitinib in patients with poor-risk mRCC are scarce and therefore, data on efficacy and safety of this therapy in this population are less known. Although patients experienced an OS of 8.16 months in this analysis, a high proportion of grade 3 or higher AEs occurred (39.6\%) and $26.4 \%$ of patients discontinued treatment due to toxicity, regardless of starting treatment at a reduced dose.

Here, we describe a median OS of 8.16 months (95\% CI, 5.73-10.59), with only $11.3 \%$ of patients exposed to second line therapy, which hi-

Figure 1 - Kaplan-Meier estimates of overall survival was 8.16 months (95\% confidence interval, 5.73-10.59).

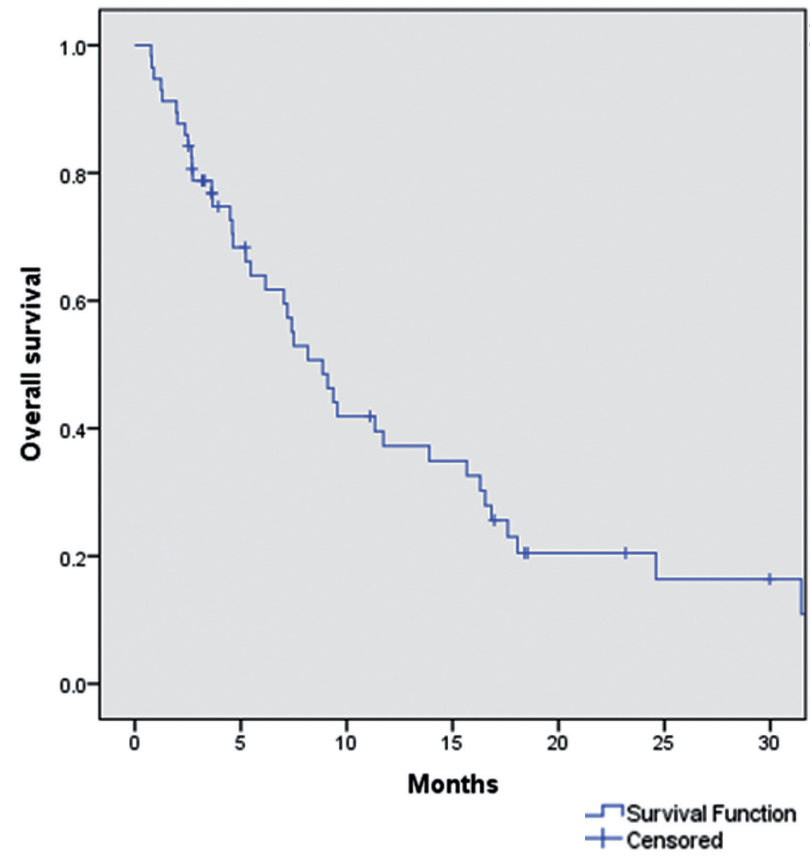

ghlights a potential survival benefit of sunitinib in the setting of poor-risk patients. This finding is consistent with the results from both the IDC original and external validation datasets demonstrated that poor-risk patients show a median OS of 8.8 and 7.8 months, respectively $(4,7)$ and compares favorably to subset analysis of phase III and IV trials $(8,9)$. In the pivotal phase III trial only $6.4 \%$ patients with poor-risk MSKCC criteria were included, rendering a median OS of 5.3 months (95\% CI: 4.2-10.0 months) with sunitinib compared with 4.0 months (95\% CI: 2.7-7.2 months) with IFN-alpha (HR: 0.660; 95\% CI: 0.360-1.207) (8). Similarly, the expanded access program (EAP) included 9\% poor-risk patients according MSKCC criteria (373 out of 4564 total subjects) and found the same median OS of 5.3 months (95\% CI: 4.6-5.4 months) (9). It must be highlighted that the numerical variations in median OS described across these different studies might be, at least in part, due to the fact of different poor risk classification, since $14 \%$ of MSKCC intermediate-risk patients are reclassified as poor-risk when stratified by IDC criteria (4).

In our series, an ORR of $17.0 \%$ was achieved and $22.6 \%$ of the evaluable patients achieved stable disease. No literature data on response rate restricted to poor risk patients is available; however, in EAP trial, which included different risk subgroups, an ORR of 17\%, was reported (9). Considering that $26.4 \%$ of our population died before response assessment, it is possible that the rate of patients who truly benefit from sunitinib is really lower. However, the lack of a predefined interval for response assessment in our retrospective series could also justify, at least in part, the lower ORR of single-agent reported here.

Other main issues addressed by our study were safety and treatment patterns. Although grade 1 and $2 \mathrm{AE}$ might have been underreported, a high proportion (39.6\%) of patients experienced grade 3 or higher AEs. In our series, the rates of grade $\geq 3$ fatigue, nausea, vomiting, and diarrhea are higher than those previously reported in both controlled and non-controlled studies (Table-4) (8-12). The high proportion of serious AE might be explained by the broader eligibility criteria. Some of the included patients had poor KPS 
Table 4 - Reported grade 3 or higher adverse events in studies evaluating sunitinib in mRCC population.

\begin{tabular}{lcccccc}
\hline Study & Type of study & Any event & Fatigue & Diarrhea & Vomiting & Nausea \\
\hline Barroso-Sousa, et al. & Retrospective & $39.6 \%$ & $15.1 \%$ & $7.5 \%$ & $7.5 \%$ & $7.5 \%$ \\
Motzer, et al. ${ }^{8}$ & Phase III & NR & $11.0 \%$ & $9.0 \%$ & $4.0 \%$ & $5.0 \%$ \\
Gore, et al. ${ }^{*}$ & Phase IV & NR & $8.0 \%$ & $5.0 \%$ & $3.0 \%$ & $2.0 \%$ \\
Chouery, et al. & Retrospective & NR & $11.0 \%$ & $7.0 \%$ & $2 \%$ & $0 \%$ \\
Porta C, et al. & Retrospective & $27.1 \%$ & $9.4 \%$ & $0 \%$ & $5.9 \%$ & $3.5 \%$ \\
Feinberg, et al. & Retrospective & $29.8 \%$ & $5.3 \%$ & $2.3 \%$ & $0.8 \%$ & $1.5 \%$ \\
\hline
\end{tabular}

mRCC: metastatic renal cell carcinoma; *current paper

(60-70), which are commonly treated under routine care, albeit excluded from randomized clinical trials (13). Furthermore, our analysis showed that 26.4\% of patients discontinued sunitinib due to $\mathrm{AE}$, which is higher than rates of 8-20\% in other studies $(9,10$, 14). In this study, 30.2\% of our patients started at the standard dose of standard dose of sunitinib. This finding might reflect the concern of AE on patients with poor KPS and older age treated with sunitinib. A recent analysis showed that approximately 30\% of elderly patients treated with sunitinib start therapy with reduced dose (15). However, in our analysis there were no were statistically significant difference in rates $\mathrm{AE}$ between these two groups of patients.

Although previous studies have examined activity and safety of sunitinib outside a clinical trial, to our knowledge, this is the first observational study that specifically addressed the efficacy, safety and treatment patterns of this treatment in a poor-risk population according IDC model. Furthermore, our data reflect a "real-world" practice setting, and OS could be accurately estimated and was consistent with that reported in the literature.

\section{CONCLUSIONS}

Our study confirms that sunitinib can be a feasible and active treatment for some patients with mRCC and poor risk features. However, the high incidence of $\mathrm{AE}$ and the drug discontinuation rates suggest a need for careful monitoring. Considering the incurable nature of advanced RCC, this retrospective study indicates that continued research efforts to identify more effective and better tolera- ted treatments for mRCC are needed, especially for poor risk patients.

\section{ACKNOWLEDGMENTS}

This work received no specific grant from any funding agency in the public, commercial, or nonprofit sectors.

\section{CONFLICT OF INTEREST}

None declared.

\section{REFERENCES}

1. Hutson TE. Targeted therapies for the treatment of metastatic renal cell carcinoma: clinical evidence. Oncologist. 2011; 16(Suppl 2): 14-22.

2. Hudes G, Carducci M, Tomczak P, Dutcher J, Figlin R, Kapoor $A$, et al. Temsirolimus, interferon alfa, or both for advanced renal-cell carcinoma. N Engl J Med. 2007; 356: 2271-81.

3. Santoni M, De Tursi M, Felici A, Lo Re G, Ricotta R, Ruggeri $\mathrm{EM}$, et al. Management of metastatic renal cell carcinoma patients with poor-risk features: current status and future perspectives. Expert Rev Anticancer Ther. 2013; 13: 697709.

4. Heng DY, Xie W, Regan MM, Harshman LC, Bjarnason GA, Vaishampayan UN, et al. External validation and comparison with other models of the International Metastatic RenalCell Carcinoma Database Consortium prognostic model: a population-based study. Lancet Oncol. 2013; 14: 141-8.

5. Common Terminology Criteria for Adverse Events (CTCAE) version 3.0. 2008. Available at http://ctep cancer gov/ protocolDevelopment/electronic_applications/docs/ctcaev3 pdf 
6. Eisenhauer EA, Therasse $P$, Bogaerts J, Schwartz LH, Sargent D, Ford R, et al. New response evaluation criteria in solid tumours: revised RECIST guideline (version 1.1). Eur J Cancer. 2009; 45: 228-47.

7. Heng DY, Xie W, Regan MM, Warren MA, Golshayan AR, Sahi $C$, et al. Prognostic factors for overall survival in patients with metastatic renal cell carcinoma treated with vascular endothelial growth factor-targeted agents: results from a large, multicenter study. J Clin Oncol. 2009; 27: 5794-9.

8. Motzer RJ, Hutson TE, Tomczak P, Michaelson MD, Bukowski RM, Oudard S, et al. Overall survival and updated results for sunitinib compared with interferon alfa in patients with metastatic renal cell carcinoma. J Clin Oncol. 2009; 27: 3584-90.

9. Gore ME, Szczylik C, Porta C, Bracarda S, Bjarnason GA, Oudard S, et al. Safety and efficacy of sunitinib for metastatic renal-cell carcinoma: an expanded-access trial. Lancet Oncol. 2009; 10: 757-63.

10. Choueiri TK, Duh MS, Clement J, Brick AJ, Rogers MJ, Kwabi $\mathrm{C}$, et al. Angiogenesis inhibitor therapies for metastatic renal cell carcinoma: effectiveness, safety and treatment patterns in clinical practice-based on medical chart review. BJU Int. 2010; 105: 1247-54.

11. Porta C, Paglino C, Imarisio I, Canipari C, Chen K, Neary M, et al. Safety and treatment patterns of multikinase inhibitors in patients with metastatic renal cell carcinoma at a tertiary oncology center in Italy. BMC Cancer. 2011; 11: 105.
12. Feinberg BA, Jolly P, Wang ST, Fortner B, Scott J, Gilmore $J$, et al. Safety and treatment patterns of angiogenesis inhibitors in patients with metastatic renal cell carcinoma: evidence from US community oncology clinics. Med Oncol. 2012; 29: 786-94.

13. Mao FJ, Rini BI. The ineligible patient: how to treat patients not included in clinical studies. World J Urol. 2014; 32: 9-18.

14. Motzer RJ, Hutson TE, Tomczak P, Michaelson MD, Bukowski RM, Rixe 0, et al. Sunitinib versus interferon alfa in metastatic renal-cell carcinoma. N Engl J Med. 2007; 356: 115-24.

15. Brunello A, Basso U, Sacco C, Sava T, De Vivo R, Camerini $A$, et al. Safety and activity of sunitinib in elderly patients $(\geq$ 70 years) with metastatic renal cell carcinoma: a multicenter study. Ann Oncol. 2013; 24: 336-42.

\section{Correspondence address:}

Romualdo Barroso-Sousa, MD

Division of Medical Oncology,

Instituto do Câncer do Estado de São Paulo (ICESP), Hospital das Clínicas da Faculdade de Medicina,

Universidade de São Paulo,

Av. Dr. Arnaldo 251, $5^{\circ}$ andar São Paulo SP, 01246-000, Brazil

Fax: +55 11 3893-2690

Email: romualdo.sousa@icesp.org.br 